Surgical

\section{management of anomalous head posture because of horizontal gaze palsy or acquired vertical nystagmus}

EC Campos, C Schiavi and C Bellusci

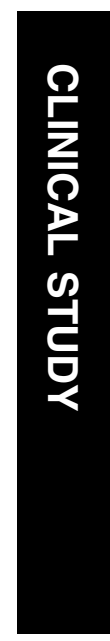

\begin{abstract}
Purpose To confirm the effectiveness of the Kestenbaum-Anderson principle in the surgical management of compensatory head posture because of horizontal gaze palsy and acquired vertical nystagmus.

Methods Nine patients with anomalous head posture because of horizontal gaze palsy, and four patients with acquired vertical nystagmus and oscillopsia and compensatory torticollis underwent surgery according to the Kestenbaum-Anderson principle. As in the treatment of congenital nystagmus, the eyes have to be shifted in the orbits, in the direction of anomalous head posture. Homonymously based prisms were used preoperatively to assess the potential benefit of surgery. At the time of surgery, the clinical conditions of the patients had been stable for at least 1 year.

Results After surgery, compensatory head posture and visual performances improved in all cases and the results remained stable for at least 2 years.

Conclusions Contrary to what is generally believed, the ocular condition of the patients with compensatory head posture secondary to neurological causes can be often improved with surgery. The aim of surgery is obviously not to modify ocular motility, but rather to improve the head position.

Eye (2003) 17, 587-592. doi:10.1038/

sj.eye. 6700431
\end{abstract}

Keywords: horizontal gaze palsy; ocular torticollis; acquired vertical nystagmus; oscillopsia; strabismus surgery
Introduction

Lesions in the brainstem, cerebellum, or cerebral hemispheres can cause supranuclear ocular motor disorders. ${ }^{1}$

Lesions of the pons, involving the paramedian pontine reticular formation (PPRF), the abducens nuclei, or both structures cause most brainstem disturbances of horizontal gaze. One-sided horizontal gaze palsy is the result of a lesion of the ipsilateral PPRF or the ipsilateral abducens nucleus. ${ }^{2}$

Destructive lesions of PPRF, such as infarction and haemorrhage, lead to a loss of all rapid eye movements towards the ipsilateral side.

Unilateral lesions of the PPRF create an ipsilateral horizontal gaze palsy similar to that produced by unilateral lesions of the abducens nucleus. ${ }^{3,4}$ In such cases, however, vestibular stimulation (oculocephalic manoeuvre or caloric stimulation) should produce complete lateral motion of the eyes and the ipsilateral abducens nucleus is presumably intact.

Lesions that produce intrinsic brainstem damage and cause acquired paralysis of horizontal gaze include trauma, ischaemia, infiltration, and compression. ${ }^{1}$

Moreover, conjugate horizontal ocular deviation may also occur with cerebellar lesions, particularly cerebellar haemorrhage. ${ }^{5}$ In such cases, the deviation is probably caused by secondary effects on the brainstem and not by cerebellar damage per se.

In total paralysis of gaze associated with lesions of the PPRF, the eyes usually remain in the midline but, particularly in acute lesions, they may deviate towards the unaffected side. The following symptoms seem to be characteristic of a chronic unilateral PPRF lesion: (1) At rest, the eyes can be held in
Ophthalmology Service University of Bologna School of Medicine Bologna, Italy

Correspondence: EC Campos Clinica Oculistica dell'Università Bologna 40138, Italy Tel: + 39051341450 Fax: + 39051342516 E-mail: campos@ alma.unibo.it

Received: 29 January 2002; Accepted: 20 August 2002

Commercial interest: None via Massarenti 9 
primary position. (2) Total gaze palsy towards the ipsilateral side. (3) Gaze preference towards the controlateral side, particularly in the partial damages of PPRF. ${ }^{6}$

Patients with horizontal gaze palsy due to PPRF damage, often reveal a secondary compensatory head posture (CHP), because they are unable to move the eyes towards the side of the palsy. These patients show a gaze preference towards the controlateral side and they adopt a CHP with the head turned to the lesion side.

Finally, acquired vertical nystagmus is a major handicap because of oscillopsia and CHP with chin-up or chin-down. ${ }^{4}$ Downbeat nystagmus is usually present in primary position. Typically, the velocity and amplitude of the upward drift are greatest on looking down and laterally: nystagmus is therefore more prominent when the patient looks in these directions. In upward gaze, downbeat nystagmus usually becomes less prominent or disappears. ${ }^{7}$ Upbeat nystagmus, present in primary position, shows similar characteristics to those of downbeat nystagmus with usually increased amplitude and frequency in upward gaze. ${ }^{8}$

Patients with acquired vertical nystagmus can assume a CHP in order to dampen their nystagmus and to reduce oscillopsia. At the present time, surgical treatment of nystagmus has been mainly limited to congenital nystagmus, not acquired nystagmus. ${ }^{9}$ Although some individual patients may be helped by retrobulbar injection of botulinum toxin, ${ }^{10,11}$ or by oral medications, ${ }^{12}$ no consistent improvement of acquired nystagmus has been reported.

Patients with such neurological ocular motility disorders are generally considered inoperable. In the last 10 years, we have performed surgery on patients with $\mathrm{CHP}$ because of horizontal gaze palsy or acquired vertical nystagmus. Obviously, the aim of surgery was not to improve ocular motility, but rather to reduce ocular torticollis. Surgery was based on the Kestenbaum-
Anderson principle, which is commonly used for the management of congenital nystagmus with the neutral zone in eccentric gaze position. ${ }^{13,14}$ This technique is directed at eliminating torticollis by shifting the eyes in the orbits in such a manner that the rest position of the nystagmus is moved to primary position. We used the same approach in acquired vertical nystagmus.

Similarly, patients with horizontal gaze palsy and CHP, with face turned to the ipsilateral side, could be helped by moving the eyes to the direction of gaze palsy. As a consequence, the head of the patients straightens.

Our preliminary results ${ }^{15}$ have been eventually confirmed in vertical acquired nystagmus with chin-up or chin-down rest position, ${ }^{16}$ and in upgaze palsy with chin-up posture. ${ }^{17}$

In the present study, we review the results obtained with the Kestenbaum-Anderson procedure in a larger group of patients (13 patients) who underwent surgery for anomalous head position because of neurological causes and followed up for a longer period of time (from 2 to 10 years). To our knowledge, this is the first extensive report with prolonged follow-up in the literature of surgical management of CHP caused by PPRF lesion or by acquired vertical nystagmus.

\section{Materials and methods}

A total of 13 consecutive patients (eight males and five females) underwent surgery to correct their CHP because of neuro-ophthalmological causes at the Department of Ophthalmology, University of Modena, Italy (between January 1989 and October 1994), and at the Ophthalmology Service of the University of Bologna, Italy (between November 1994 and January 1999).

Nine patients presented horizontal gaze palsy and four cases showed acquired vertical nystagmus with oscillopsia. Pertinent data on these patients are summarized in Table 1.

Table 1 Materials

\begin{tabular}{rclll}
\hline Patient & Age (years) & Condition & CHP (deg) & Aetiology of gaze disorders \\
\hline 1 & 66 & L.H.g.p. & $20^{\circ}$ left & Pontine ischaemia \\
2 & 38 & L.H.g.p. & $20^{\circ}$ left & Head trauma \\
3 & 69 & L.H.g.p. & $25^{\circ}$ left & Pontine infarction \\
4 & 67 & R.H.g.p. & $18^{\circ}$ right & Brainstem haemorrhage \\
5 & 72 & R.H.g.p. & $20^{\circ}$ right & Pontine haemorrhage \\
6 & 35 & R.H.g.p. & $15^{\circ}$ right & Head trauma \\
7 & 35 & L.H.g.p. & $15^{\circ}$ left & Brainstem tumour \\
8 & 65 & L.H.g.p. & $27^{\circ}$ left & Cerebellar haemorrhage \\
9 & 42 & R.H.g.p. & $18^{\circ}$ right & Brainstem tumour \\
10 & 55 & Acq.vert.ny. & $25^{\circ}$ chin-down & Brainstem tumour \\
11 & 33 & Acq.vert.ny. & $30^{\circ}$ chin-down & Head trauma \\
12 & 58 & Acq.vert.ny. & $25^{\circ}$ chin-up & Brainstem infarction \\
13 & 35 & Acq.vert.ny. & $35^{\circ}$ Chin-down & Arnold Chiari syn. \\
\hline
\end{tabular}

CHP: compensatory head posture; H.g.p.: horizontal gaze palsy (R: right, L: left); Acq.vert.ny.: acquired vertical nystagmus. 
We excluded from the study patients with horizontal gaze palsy that revealed at neurological and electromyographic examination abducens nucleus damage. Usually, these patients showed esotropia ipsilateral to horizontal gaze palsy, and CHP was required in order to obtain single binocular vision rather than as a consequence of a conjugate gaze palsy. ${ }^{18}$

At the time of extraocular muscles surgery, the clinical picture had been stable for at least 12 months. The age at surgery ranged from 33 to 72 years (mean, 52 years). The average of follow-up was 4 years and 9 months with a range of 2-10 years.

Preoperative and postoperative CHP was measured with the patient fixating on the smallest visual acuity line that he/she could read at distance $(6 \mathrm{~m})$ under binocular viewing conditions. In this study, we considered a CHP clinically significant if the following criterion for each orientation was met: a face turn of $15^{\circ}$ or more and chin-up or chin-down of $10^{\circ}$ or more. ${ }^{19}$

A simple surgical goniometer with graded markings, commonly used by orthopaedic surgeons for measurement of join angles and scoliosis, was found to be excellent for measuring $\mathrm{CHP}^{20}$ We used such ruler to calculate head turn and vertical plane torticollis, preoperatively and at any time of follow-up. CHP was measured by different examiners who were masked to the patient's clinical history and whether the measurement was taken before or after surgery. While there is little need for such measurements in clinical practice where pre- and postoperative photographs are sufficient for documentation, such instrument is useful in quantitating the effect of surgical procedure on abnormal head position.

Best-corrected visual acuity (evaluated with Snellen Es) was measured under binocular conditions at distance $(6 \mathrm{~m})$ and near fixation $(33 \mathrm{~cm})$, both in the position of torticollis and after surgical correction of CHP. In acquired nystagmus patients, preoperative visual acuity was measured either in primary position or with CHP when nystagmus appeared to be less evident. The same chart was used preoperatively and postoperatively.

Homonymously based prisms were preoperatively used to assess the potential benefit of surgery. In fact, like for congenital nystagmus patients, an improvement in head position with the use of homonymous prisms is an essential prerequisite for surgery. The prisms are inserted with the base opposite the direction of gaze. For instance, with a head turn to the left because of a left horizontal gaze palsy, a prism base-in before the right eye and baseout before the left eye will correct the head turn. The results of surgery can be reasonably well predicted on the basis of the patient's response to homonymous prisms.

In two complex cases (patient no. 3,8), mepivacainum cloridratum $2 \%$ was injected preoperatively under electromyographic control into the left medial rectus and right lateral rectus muscles in order to determine if surgery would be useful. Having obtained a temporary improvement in head position, surgery was then successfully performed at a later date.

Surgery was based on the Kestenbaum-Anderson principle of rotating the eyes in the orbits, used for congenital nystagmus. ${ }^{13,14,21}$ The eyes have to be shifted in the direction of anomalous head posture. In cases where the lateral gaze position was highly eccentric, that is, more than $20^{\circ}$, large recessions (enhanced-Anderson operation) were performed on the agonist horizontal muscles (12-14 $\mathrm{mm}$ for the lateral rectus muscle in one eye and $10-12 \mathrm{~mm}$ for the medial rectus muscle in the other eye). For instance, in the presence of $20^{\circ}$ face turn to the left, the right lateral rectus muscle was recessed $12 \mathrm{~mm}$ and the left medial rectus muscle was recessed $10 \mathrm{~mm}$. Less severe torticollis were treated with smaller amounts of recession.

In the presence of acquired nystagmus with dampening of the jerks in up-gaze or down-gaze and compensatory chin-down or chin-up position, respectively, we performed large recessions of both superior or inferior rectus muscles $(8-10 \mathrm{~mm})$. Videotapes were obtained before and after surgery. Electro-oculography was not performed.

All patients of this study had informed consent about the aim of surgery and if there was medical contraindication for general anaesthetic, the surgery was performed under local anaesthetic.

\section{Results}

All patients presented preoperative medium- to highgrade CHP (mean $23^{\circ}$, standard deviation (SD) $6^{\circ}$ ).

After surgery, CHP and visual comfort improved in all cases and the results remained stable for at least 2 years. Compensatory head position improved with mean residual torticollis of $6^{\circ}\left(\mathrm{SD} 4^{\circ}\right)$ at the maximum followup (Table 2).

Small amount of CHP was present in most patients and hypercorrection was never observed at any time of the study (Figure 1). Moderate reduction of the surgical outcome was noted in patients with longer follow-up; four out of 13 patients showed a small shift back to the original head position. Anyway, patients revealed a CHP larger than the criterion that we considered as success of treatment.

Occasional but temporary diplopia during the immediate postoperative phase, because of fusion difficulties, was referred in four patients (30\%). No patient complained double vision 1 month after extraocular muscle surgery. Two out of nine patients with horizontal gaze palsy (patients no. 4 and 8) obtained only 
Table 2 Results

\begin{tabular}{|c|c|c|c|c|}
\hline \multirow{2}{*}{$\frac{\text { Patient }}{1}$} & \multicolumn{2}{|c|}{ Yoke muscles recession ( $\mathrm{mm}$ ) } & \multirow{2}{*}{$\frac{\text { Residual CHP (deg) }}{5^{\circ}}$} & \multirow{2}{*}{$\frac{\text { Follow-up (years) }}{3}$} \\
\hline & LMR (8) & RLR (10) & & \\
\hline 2 & LMR (9) & RLR (11) & $5^{\circ}$ & 7 \\
\hline 3 & LMR (10) & RLR (12) & $10^{\circ}$ & 10 \\
\hline \multirow[t]{2}{*}{4} & RMR (9) & LLR (11) & $10^{\circ}$ & 3 \\
\hline & \multicolumn{2}{|c|}{ (+ resection of antagonist RLR, LMR) } & & \\
\hline 5 & RMR (9) & LLR (11) & $3^{\circ}$ & 5 \\
\hline 6 & RMR (6) & LLR (7) & $0^{\circ}$ & 8 \\
\hline 7 & LMR (6) & RLR (7) & $0^{\circ}$ & 2 \\
\hline \multirow[t]{2}{*}{8} & LMR (12) & RLR (14) & $15^{\circ}$ & 3 \\
\hline & \multicolumn{2}{|c|}{ (+ resection of antagonist LLR, RMR) } & & \\
\hline 9 & RMR (8) & LLR (10) & $3^{\circ}$ & 3 \\
\hline 10 & RSR (8) & LSR (8) & $5^{\circ}$ & 8 \\
\hline 11 & RSR (10) & LSR (10) & $5^{\circ}$ & 6 \\
\hline 12 & RIR (8) & LIR (8) & $5^{\circ}$ & 2 \\
\hline 13 & RSR (10) & LSR (10) & 10 & 3 \\
\hline
\end{tabular}

CHP: compensatory head posture; MR: medial rectus muscle; LT: lateral rectus muscle; SR: superior rectus muscle; IR: inferior rectus muscle (R: right, L: left).
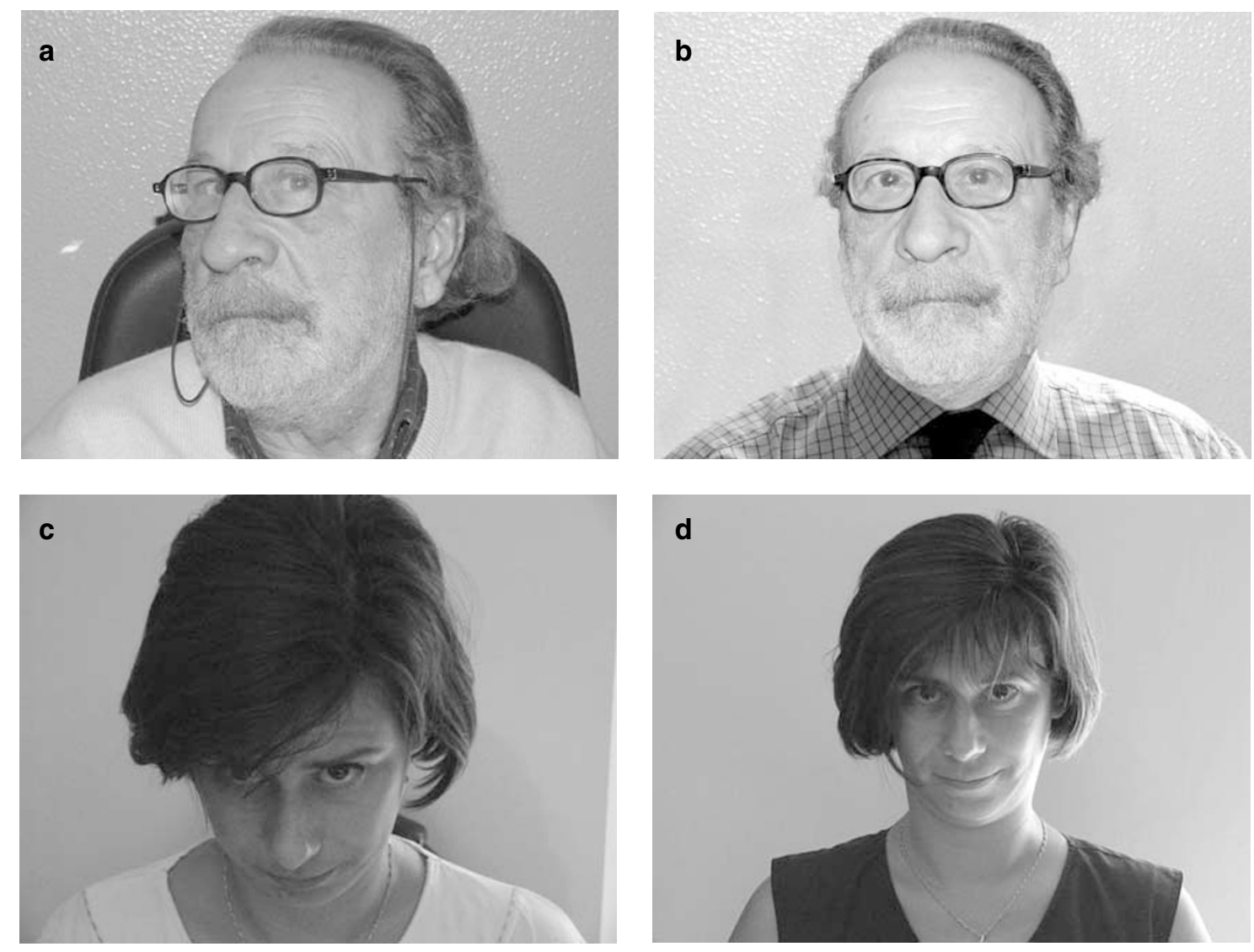

Figure 1 Patient with right horizontal gaze palsy and head turn of approximately $20^{\circ}$ to the right (a); the same patient 1 year after recession of right medial rectus and left lateral rectus muscles (b). Note: the patient can use his glasses more effectively. Patient with acquired nystagmus equilibrium in upward gaze; $\mathrm{CHP}$ with chin-down is present (c); the same patient 1 year after surgical weakening of both superior rectus muscles (d).

partial reduction of CHP as a result of the first surgery. They required secondary surgical resection of antagonistic horizontal rectus muscles 6 months later.
The patients with acquired nystagmus and vertical plane torticollis showed an excellent improvement of CHP (Figure 1c and d). The shift of the zone of 
nystagmus equilibrium approximately in primary position was achieved in all cases (CHP smaller than $10^{\circ}$ ). The patient with chin-up posture (no. 12) revealed postoperatively a slight narrowing of the palpebral fissure as a result of the large weakness of the inferior recti.

Improvement of visual acuity in primary gaze was seen in all four patients with acquired nystagmus. Mean preoperative best-corrected visual acuity measured in primary position was 20/80. Postoperative best-corrected visual acuity measured with the same settings improved to 20/30 in primary gaze with better head posture. Similarly, mean postoperative visual acuity at near fixation improved from $20 / 40$ to $20 / 25$.

\section{Discussion}

Most common ocular causes of CHP include congenital nystagmus, strabismus, blepharoptosis, and uncorrected refractive errors. ${ }^{18}$ Anyway, supranuclear ocular motility disturbances, such as horizontal gaze palsy or acquired vertical nystagmus, can cause an abnormal $\mathrm{CHP}$.

The results of our study confirm that an improvement of visual comfort is possible with extraocular muscles surgery for patients with complex ocular motility disorders, whenever these conditions cause an ocular torticollis. A careful neuro-ophthalmological evaluation is necessary to clearly define the type of oculomotor disorder present and to accurately predict the advantages of surgery. Moreover, surgery should be taken into account only if neurological condition appears clearly stable.

Vascular diseases were the most frequent causes involved in our neurological origin CHP. Head trauma was found in three patients. Most of the reported cases of ocular dysmotility from brainstem trauma recovered completely; this is in contrast to the permanent deficit secondary to pure vascular disease of brainstem. ${ }^{22}$

CHP yields a number of possible advantages for patients with horizontal gaze palsy or acquired nystagmus. Patients with unilateral lesion of PPRF show a gaze preference towards the controlateral side with $\mathrm{CHP}$ mostly at distance fixation. On the contrary, CHP disappears at near fixation because they may substitute convergence for impaired conjugate adduction and then crossfixate to extend their range of view.

Particular care must be taken when looking for CHP in patients with acquired vertical nystagmus and oscillopsia. ${ }^{23}$ The compensatory gaze position often exists: its excessive eccentricity makes it however unusable for the patient (Figure 1c).

The aim of extraocular muscles surgery is obviously not to improve ocular motility, but rather to improve CHP. Surgery is indicated only when head position improvement is observed using homonymously based prisms preoperatively. Whenever there are doubts as to the usefulness of surgery, local anaesthetic can be injected under electromyographic control to determine the effect of chemorecession of a pair of yoke muscles on the ocular torticollis. We applied such diagnostic test in two high-grade CHP cases.

In the presence of horizontal gaze palsy, we recessed the yoke horizontal muscles in both eyes as first surgical choice. Since recession is more effective on the medial rectus than on the lateral rectus, lesser amounts of recession were used on the medial rectus, in order not to induce exodeviations. Patients whose CHP resulted highly undercorrected after yoke horizontal muscles recession underwent resection of antagonistic rectus muscles in a second procedure (patients no. 4 and 8).

Adjustable sutures were never used in our patients because in the absence of strabismus, conventional recession or unconventionally large recession (up to $14 \mathrm{~mm}$ : super-Anderson procedure) of the yoke muscles is safe and effective in eliminating or reducing CHP and there is no risk of inducing jatrogenic strabismus. On the other hand, adjustable sutures are useful in the surgical management of CHP when gaze palsy or nystagmus is accompanied by strabismus. ${ }^{17}$

Most of our patients with ocular torticollis because of horizontal gaze palsy showed undercorrection of anomalous head position. In fact, some patients with long-standing head postures will, despite successful treatment of the underlying condition, continue to demonstrate a tendency to $\mathrm{CHP}^{24}$ These are usually attributed to alterations in the tone of the neck muscles that may not reverse even if the ocular causes are rectified. Nevertheless, postoperative residual head turn may be alleviated further with low-power prisms. In this way, watching television can be made easier and more comfortable; walking ability can be improved as well as the general way of life of the patient. Moreover, cosmetic improvement was unquestionable.

Visual acuity both at distance and at near fixation improved in most of our patients. In fact, eliminating the $\mathrm{CHP}$ in the presence of refractive errors can lead to visual acuity improvement because of a more effective use of glasses (Figure 1a, b). Also, in acquired vertical nystagmus an improvement of both oscillopsia and visual acuity can be obtained with surgery. An eccentric gaze position can exist where oscillopsia is reduced or absent altogether. Often, such gaze position is so eccentric as to be useless for the patient. With surgery, the zone of nystagmus equilibrium is shifted to the primary position (Figure 1c, d).

Our results indicate that even patients with torticollis because of gaze palsy or acquired nystagmus benefit from surgery. After stabilization of their neurological 
condition, there is no sense to wait for possible spontaneous improvement of $\mathrm{CHP}$, which will not take place. The purpose of this work is to promote a larger use of oculomotor surgery in horizontal gaze palsy and in acquired vertical nystagmus.

\section{References}

1 Miller NR, Newman NJ. Walsh and Hoyt's Clinical NeuroOphthalmology, 5th ed. Lippincott Williams \& Wilkins: Baltimore, MD, 1999.

2 Daroff RB, Troost BT. Supranuclear disorders of eye movements. In: Duane TD, Jager EA (eds). Clinical Ophthalmology, Vol 2. Harper and Row: Philadelphia, Chapter 10, 1987.

3 Henn V, Lang W, Hepp K, Reisine H. Experimental gaze palsies in monkeys and their relation to human pathology. Brain 1984; 107: 619-636.

4 Leigh RJ, Zee DS. The neurology of eye movement, 3rd ed. Oxford University Press Inc.: New York, NY, 1999.

5 Daroff RB, Hoyt WF. Supranuclear disorders of ocular control system in man: clinical, anatomical and physiological correlations. In: Back-y-Rita P, Collins CC, Hyde JE (eds). The Control of Eye Movements. Academic Press: New York, 1971, pp 175-235.

6 Henn V, Buttner U. Disorders of horizontal gaze. In: Lennerstrand G, Zee DS, Keller EL (eds). Functional Basis of Ocular Motility Disorders. Pergamonn Press Ltd: Oxford, 1982, pp 239-245.

7 Halmagyi GM, Rudge P, Gresty MA, Sanders MD. Downbeating nystagmus. A review of 62 cases. Arch Neurol 1983; 40: 777-784.

8 Fisher A, Gresty M, Chambers B, Rudge P. Primary position upbeating nystagmus. A variety of central positional nystagmus. Brain 1983; 106(4): 949-964.

9 Lee JP. Surgical management of nystagmus. Eye 1988; 2: 44-47.

10 Helveston EM, Pogrebniak AE. Treatment of acquired nystagmus with botulinum A toxin. Am J Ophthalmol 1988; 106: $584-586$
11 Tomsak RL, Remler BF, Averbuch-Heller L, Chandran M, Leigh RJ. Unsatisfactory treatment of acquired nystagmus with retrobulbar injection of botulinum toxin. Am J Ophthalmol 1995; 119: 489-496.

12 Averbuch-Heller L, Tusa RJ, Fuhry L, Rottach KG, Ganser GL, Heide $\mathrm{W}$ et al. A double-blind controlled study of gabapentin and baclofen as treatment for acquired nystagmus. Ann Neurol 1997; 41(6): 818-825.

13 Anderson JR. Causes and treatment of congenital eccentric nystagmus. Br J Ophthalmol 1953; 37: 267.

14 Kestenbaum A. Nouvelle opération du nystagmus. Bull Soc Ophtalmol Fr 1953; 6: 599.

15 Schiavi C, Scorolli L, Campos EC. Surgical management of anomalous head posture due to supranuclear gaze palsies and acquired nystagmus. In: Spiritus M (ed). Transactions of the 23rd Meeting E.S.A.; Nancy. 1996, pp 229-232.

16 Spielmann A, Aflalo G, Spielmann AC. Surgery in vertical acquired nystagmus. In: Lennerstrand G (ed). Advances in Strabismology. Aeolus Press: Buren 1999, pp 369-372.

17 Buckley SA, Elston J. Surgical treatment of supranuclear and internuclear ocular motility disorders. Eye 1997; 11: 377-380.

18 Von Noorden GK, Campos EC. Binocular Vision and Ocular Motility: Theory and Management of Strabismus, 6th ed. Mosby: St Louis, 2002.

19 Kraft SP, O'Donoghue EP, Roarty JD. Improvement of compensatory head posture after strabismus surgery. Ophthalmology 1992; 99: 1301-1308.

20 Mitchell PR, Wheeler MB, Parks MM. Kestenbaum surgical procedure for torticollis secondary to congenital nystagmus. J Pediatr Ophthalmol Strabismus 1987; 24: 87-93.

21 Decker W de. Die chirurgische Behandlung der Kopfblickzwangshaltungen. Buch Augenarztes 1984; 99: 300.

22 Baker RS, Epstein AD. Ocular motor abnormalities from head trauma. Surv Ophthalmol 1991; 35(4): 245-267.

23 Von Noorden GK, Munoz M, Wong SY. Compensatory mechanism in congenital nystagmus. Am J Ophthalmol 1987; 104: 387-397.

24 Morris JE. Head posturing in horizontal strabismus. Am Orthopt J 1983; 33: 32-35. 